\title{
Highly tunable magnetic coupling in ultrathin topological insulator films due to impurity resonances
}

\author{
Mahroo Shiranzaei, Jonas Fransson, Annica M. Black-Schaffer, and Fariborz Parhizgar (1) \\ Department of Physics and Astronomy, Uppsala University, Box 516, S-751 20 Uppsala, Sweden
}

(Received 11 March 2020; accepted 11 November 2020; published 30 November 2020)

\begin{abstract}
We theoretically investigate the carrier-free exchange interaction between magnetic impurities in ultrathin $\mathrm{Bi}_{2} \mathrm{Se}_{3}$ topological insulator films by taking into account the low-energy states produced by the impurities. To match with experimental observations of magnetism on the surface of ultrathin topological insulator films, we restrict the calculations to having the chemical potential within the energy gap, with then interband processes mediating the exchange interaction. We find that the locally induced impurity resonances strongly influence the exchange interaction between magnetic moments. In particular, we find a noncollinear alignment to be more favorable than the collinear ferromagnetic alignment preferred when impurity states are ignored and only the pristine topological insulator band structure is considered. As a result, chiral ferromagnetism can easily become favorable over the ferromagnetic phase in these materials. Moreover, we show that by applying an electric field perpendicular to the ultrathin film, the exchange interaction can be drastically enhanced. This generates the possibility of highly tunable magnetism by electric field.
\end{abstract}

DOI: 10.1103/PhysRevB.102.174446

\section{INTRODUCTION}

In the absence of direct coupling, magnetic impurity moments in solids couple indirectly to each other through the electronic band of the host material, with the nature of the coupling mechanism then determined by the electronic structure of the host. In terms of spatial extension, magnetic moments couple at distances up to a few nanometers in topological insulators (TIs), whereas the corresponding coupling in most semiconducting materials ranges only at most a few $\AA$ [1]. This long-range coupling between the magnetic impurities in topological insulators leads to the existence of a robust magnetic phase, which has also been recently confirmed in experiments, both using angular resolved photoemission spectroscopy (ARPES) and scanning tunneling microscopy (STM) [2-4].

It has been suggested that the indirect magnetic coupling in topological insulators is governed by topology [2]. While the magnetically ordered phase in topologically trivial materials is fragile to decreasing dimensionality, the magnetic phase in topological insulators appears to be strengthened when lowering the dimension. Indeed, it has been observed that the magnetic phase generated by coupled magnetic impurities on the surface of topological insulators is far more robust than the corresponding phase created between bulk impurities [5]. In line with this, magnetic coupling of impurities has also been verified in ultrathin films of topological insulators in

Published by the American Physical Society under the terms of the Creative Commons Attribution 4.0 International license. Further distribution of this work must maintain attribution to the author(s) and the published article's title, journal citation, and DOI. Funded by Bibsam. experiments measuring the quantum anomalous Hall effect (QAHE) [6-10]. One prerequisite for the QAHE is the existence of an energy band gap, see, e.g., Ref. [10], thus being relevant only in topological insulator thin films, as for films thinner than five quintuple layers, the surface states become gapped due to hybridization between the surface states at opposite sides [11]. The finite energy gap in such ultrathin topological insulator films however also makes the possible emergence of impurity resonances within the band gap a highly sensitive topic, as that can then easily change the conditions for the QAHE in a discontinuous way. In addition, with the band dispersion of ultrathin topological insulator films tunable by the application of an electric field, a tantalizing electric control of magnetism [12] might also be achievable in the ultrathin limit.

Very generally, the indirect magnetic coupling between magnetic impurities is governed by the magnetic susceptibility of the whole system. Thus, in materials with strong spin-orbit interaction, such as topological insulators, the magnetic susceptibility tensor gives three fundamentally different contributions: an isotropic (Heisenberg-like), a symmetrically anisotropic (Ising-like), and an asymmetrically anisotropic (Dzyalosinskii-Moryia-like) contribution [13-15]. The isotropic and symmetrically anisotropic contributions lead to collinear configurations of the magnetic moments, whereas the asymmetrically anisotropic contribution favors noncollinear configurations [16]. This asymmetric anisotropy easily leads to the existence of exotic magnetic phases, such as spin-glass, or chiral ferro- and antiferromagnetic phases [17,18].

Furthermore, the nature of the indirect coupling between magnetic impurities is significantly different between metallic and gapped materials. The interaction in metals is dominated by excitations around the Fermi level, known as RudermanKittel-Kausya-Yosida (RKKY) interaction, with intraband 
processes giving the primary contribution to the magnetic susceptibility. On the other hand, in gapped systems, where the chemical potential intersects no bands, the intraband process vanishes and the coupling is necessarily provided by interband processes between the valence and conduction band electrons, more related to van Vleck-type magnetism. In fact, van Vleck magnetism based on interband susceptibility has already been observed in topological insulator thin films [19-21]. With the indirect coupling determined by the magnetic susceptibility of the whole system, the impurities themselves also modify the indirect coupling $[16,22,23]$. In particular, in topological insulators, impurity resonance states easily appear near the Dirac point due to scattering off the nonmagnetic part of the impurity potential [24-26]. In ultrathin topological insulator films these resonances may even arise inside the band gap, depending on the strength of the impurity scattering potentials and the properties of the topological insulator [24,27], also recently addressed using $a b$ initio calculations $[19,23,28]$. The gapped dispersion in ultrathin topological insulator films thus makes impurity states even more prominent as they can be the only low-energy states available to mediate the indirect magnetic coupling. Although bulk doping of topological insulators, including the effects of impurities, has been numerically studied [29], most calculations of the magnetic susceptibility in topological insulator systems (and even other structures such as gapped graphene [30], $\mathrm{MoS}_{2}$ [31], bilayer graphene [32]) have been based only on the itinerant electrons of the unperturbed, or pristine, TI surface states $[21,33]$. Only recent work highlighting the effect of the impurities, and then only in thick topological insulators, where it is the RKKY interaction mediating the coupling [16]. This is in contrary to the fact that recent experiments on ultrathin topological insulator films have indicated that the indirect magnetic coupling depends strongly on the nature of the magnetic impurities [1,2,6-9].

In this work, we therefore calculate the influence on the magnetic susceptibility of the impurity induced in-gap resonances in ultrathin topological insulator films. To accurately capture the indirect coupling between surface magnetic impurity moments, we use the $T$-matrix approach which treats the effects of the impurities to infinite order in perturbation theory. Being a gapped system, RKKY interaction is not present and hence we focus on the impact of impurity states on the interband van-Vleck magnetic susceptibility. We show that the impurity resonances quench both the isotropic Heisenberg and symmetrically anisotropic Ising contributions. Moreover, we find a strong energy dependence, which opens for excellent tuning possibilities of the indirect coupling on- and offresonance. Most importantly, we find that the asymmetrically anisotropic Dzyalosinskii-Moryia contribution is generated when we include the impurity states. As a direct consequence, a noncollinear collective configuration of the magnetic moments easily becomes favored, with a finite out-of-plane net magnetization. This is in sharp contrast to the ferromagnetic ground state previously obtained when only considering the pristine ultrathin topological insulator films [21]. Finally, we also show that by simply applying an external electric field, the magnetic coupling becomes extensively tunable, between ferro- and antiferromagnetic to chiral configurations. This tunability spans order of magnitudes and is due to the impurity states, which is different from the minor electrical tunability reported for magnetic coupling in other systems [21,32,33].

The remainder of the paper is organized as follow. In Sec. II, we introduce the model Hamiltonian and the general formalism for calculating the magnetic susceptibility, including the impurity states. In Sec. III we present our results, with a focus on the contribution from impurity resonances and their tunability. Finally, we summarize and offer a few concluding remarks in Sec. IV. Some detailed part of the calculations can be found in Appendices A-F.

\section{MODEL AND METHOD}

The low-energy properties of the surface state electrons in the ultrathin topological insulator films can be described by an effective two-dimensional Hamiltonian near the $\Gamma$ point describing the two surfaces $[11,34]$

$$
\mathcal{H}_{0}(\mathbf{k})=\tau_{z} \otimes\left[\hbar v_{F}(\mathbf{k} \times \hat{\mathbf{z}}) \cdot \boldsymbol{\sigma}+V \sigma_{0}\right]+\Delta \tau_{x} \otimes \sigma_{0}-\mu .
$$

Here, $\boldsymbol{\sigma}, \boldsymbol{\tau}$ are Pauli matrices in the spin and surface space, respectively, $\mathbf{k}=\left(k_{x}, k_{y}\right)$ denotes the two-dimensional wave vector for the surface electrons, and $v_{F}$ is the Fermi velocity. Moreover, $V$ denotes the potential difference between the two surfaces. This potential arises from the effect of a substrate and/or an external electric field applied perpendicular to the film. Due to the thickness of the film there is an effective mass hybridization term of the form $\Delta \equiv \delta_{0}-\delta_{1} k^{2}$ that couples the two surfaces of the topological insulator. The sign changing $\delta_{1}$ term is responsible for a topological phase transition [34], bringing the system into a quantum spin Hall state with gapless edge modes. However, since our study is restricted both to the bulk effects of topological insulator thin films without consideration of the one-dimensional edges and to low energy chemical potentials, this higher expansion $k$ term can be safely neglected [35] and hence we assume $\Delta=\delta_{0}$. For more details, please see Appendix D.

The model in Eq. (1) leads to the dispersion relation

$$
E_{s m}(k)=s \sqrt{\left(\hbar v_{F}|\mathbf{k}|+(-1)^{m} V\right)^{2}+\Delta^{2}},
$$

of a gapped Dirac spectrum with gap size $2 \Delta$. Here, $s= \pm 1$, refers to the conduction and the valence bands, respectively, whereas $m=1,2$ labels the solutions. Further, we model the added magnetic impurities, which modify the electronic structure of the system, by the Hamiltonian

$$
\mathcal{H}=\sum_{i}\left(U \sigma_{0}+J_{c} \mathbf{S}_{i} \cdot \boldsymbol{\sigma}\right) \delta\left(\mathbf{r}-\mathbf{r}_{i}\right),
$$

where $U$ and $J_{c} \mathbf{S}_{i}$ represent the spin-independent and spindependent scattering potentials of the impurity, respectively. For sufficiently large scalar potential $U$, impurity-induced resonance states emerge inside the gap, while the spin scattering off $J_{c} \mathbf{S}_{i}$ breaks the spin degeneracy of these states. The energies of the impurity resonances depend inversely on the scattering potential $U$, approaching the middle of the gap (Fermi energy) as $U \rightarrow \infty$, whereas the spin splitting of the resonances is governed by $J_{c} \mathbf{S}_{i}$. Here we allow magnetic impurities on sites $\mathbf{r}_{i}$, summing over all impurities, where we restrict ourselves to consider impurities in the top surface only, 
justified by several experiments on magnetism on the surface of ultrathin topological insulator films due to the surface impurities [1-5]. The only assumption in Eq. (3) is that the impurities behave as classical spins, i.e., $J_{c} \hat{S}=J_{c}\langle S\rangle \equiv J_{c} \mathbf{S}$, with strength $\left|\mathbf{S}_{i}\right|=S$, as appropriate for higher spin impurities [22].

For two local impurity magnetic moments, located at $\mathbf{r}$ and $\mathbf{r}^{\prime}$, respectively, the effective indirect coupling, or exchange, Hamiltonian can be written as

$$
\mathcal{H}^{\mathrm{ex}}=\frac{1}{2} J_{c}^{2} \mathbf{S}_{1} \cdot \chi\left(\mathbf{r}, \mathbf{r}^{\prime}\right) \cdot \mathbf{S}_{2},
$$

where $\chi\left(\mathbf{r}, \mathbf{r}^{\prime}\right)$ is the magnetic susceptibility tensor. This tensor can be obtained from the definition of the magnetization in terms of the dressed Green's functions in a full expansion of the impurity perturbation by using the $T$-matrix approach [16]. The result we can always write $\chi\left(\mathbf{r}, \mathbf{r}^{\prime}\right)$ as

$$
\chi\left(\mathbf{r}, \mathbf{r}^{\prime}\right)=\operatorname{tr} \int \frac{\sigma \mathcal{G}_{0}\left(\mathbf{r}, \mathbf{r}^{\prime} ; \omega\right) \boldsymbol{\sigma} \mathcal{G}_{0}\left(\mathbf{r}^{\prime}, \mathbf{r} ; \omega\right)}{(1-U g)^{2}-J_{c}^{2} S^{2} g^{2}} \frac{d \omega}{2 \pi} .
$$

This expression describes the indirect exchange interaction between two impurities at some finite distance $\mid \mathbf{r}-$ $\mathbf{r}^{\prime} \mid \neq 0$. In this expression, $\mathcal{G}_{0}\left(\mathbf{r}, \mathbf{r}^{\prime} ; \omega\right)$ denotes the bare single electron Green's function, i.e., without impurities, the trace runs over the spin degrees of freedom, $g(\omega)=$ $\left(1 / k_{c}^{2}\right) \operatorname{tr} \int \mathcal{G}_{0}(\mathbf{k}, \omega) d \mathbf{k} / 2$, and where the expression in the denominator encode for the contributions from the impurity states. The normalization factor $k_{c}$, in the definition of $g(\omega)$, is the cutoff band momentum and ensures that $g$ and $\mathcal{G}_{0}$ have the same dimension. For sufficiently weak scattering potentials, the impurity resonances emerge within the valence and conduction bands, that is, outside the gap. In this regime the mechanism for spin-spin interaction can be described sufficiently well within linear response theory. Hence, the magnetic susceptibility of the pristine topological insulator, $\chi_{0}$, is retained by setting $U=J_{c} S=0$ in the denominator. We refer to Appendix A for more details on the bare Green's function. We note that Eq. (4) is the same as found in Ref. [16], as it expresses the most general form of the magnetic susceptibility for a system with magnetic impurities, although the purpose and setup of this previous work were much different from here. We also already here see that Eq. (5), in combination with the highly nontrivial expressions for the Green's function, results in a magnetic susceptibility that is not easily accessible analytically but that a numerical solution is needed. We also note that usually when treating the spin as a quantum object, we need to be careful, since $\langle S\rangle^{n} \neq\left\langle S^{n}\right\rangle$ in general for integers $n>1$. However, as the numerator of Eq. (5) is only proportional to $\langle S\rangle$ and the denominator captures the existence of impurity states seen in experiment, we expect that any quantum effects of the spin can for our purposed be omitted without loosing information on the qualitative behavior of the interaction. It is convenient to rotate the spin vectors $\mathbf{S}_{i=1,2}$ into $\tilde{\mathbf{S}}_{i}=\left(S_{i x} \cos \varphi_{R}, S_{i y} \sin \varphi_{R}, S_{i z}\right)[16,33]$ in terms of the polar angle $\varphi_{R}$ of the relative distance between the impurities. Then the exchange Hamiltonian takes the form

$$
\begin{aligned}
\mathcal{H}^{\mathrm{ex}}= & {\left[H \mathbf{S}_{1} \cdot \mathbf{S}_{2}+I\left(\tilde{\mathbf{S}}_{1} \cdot \tilde{\mathbf{S}}_{2}+\tilde{S}_{1 x} \tilde{S}_{2 y}+\tilde{S}_{1 y} \tilde{S}_{2 x}\right)\right.} \\
& \left.+\mathbf{D} \cdot\left(\tilde{\mathbf{S}}_{1} \times \tilde{\mathbf{S}}_{2}\right)\right] .
\end{aligned}
$$

Here $H$ and $I$ refer to the isotropical and symmetrical anisotropic couplings, respectively, whereas $\mathbf{D}=D(1$, $-1,0)$ denotes the asymmetrical anisotropy. Here $H, I$, and $\mathbf{D}$ can be thought of as Heisenberg-, Ising-, and DzyaloshinskiiMoriya-like interactions, respectively. Using Eq. (5) for the magnetic susceptibility in Eq. (4), these interaction parameters are obtained from the expressions [16]

$$
\begin{aligned}
H & =J_{c}^{2} \int \frac{\mathcal{G}_{t t}^{2}\left(\mathbf{r}, \mathbf{r}^{\prime} ; \omega\right)+\mathcal{G}_{t t}^{2}\left(\mathbf{r}, \mathbf{r}^{\prime} ; \omega\right)}{(1-U g)^{2}-J_{c}^{2} S^{2} g^{2}} \frac{d \omega}{\pi}, \\
I & =-2 J_{c}^{2} \int \frac{\mathcal{G}_{t t}^{2}\left(\mathbf{r}, \mathbf{r}^{\prime} ; \omega\right)}{(1-U g)^{2}-J_{c}^{2} S^{2} g^{2}} \frac{d \omega}{\pi}, \\
D & =-2 J_{c}^{2} \int \frac{\mathcal{G}_{t t}\left(\mathbf{r}, \mathbf{r}^{\prime} ; \omega\right) \mathcal{G}_{t t}\left(\mathbf{r}, \mathbf{r}^{\prime} ; \omega\right)}{(1-U g)^{2}-J_{c}^{2} S^{2} g^{2}} \frac{d \omega}{\pi} .
\end{aligned}
$$

Here $\mathcal{G}_{t t}$ and $\mathcal{G}_{t t}$ indicate the $\uparrow \uparrow$ and $\uparrow \downarrow$ components, respectively, of the bare Green's function on the top surface, see Appendix A for more details. Again, the results for a pristine topological insulator, $J_{0}=H_{0}, I_{0}, D_{0}$, is obtained by setting $J_{c} S=U=0$ in the denominator of Eq. (7). In order to provide a physical understanding for the behavior of the exchange parameters in the presence of impurities, we also consider the spin-polarized local density of states (spin-resolved LDOS) $\rho_{\uparrow, \downarrow}$, see Appendix B for a detailed expression derived from the full Green's function.

Before proceeding we also note that the magnetic susceptibility is, in general, a sum of interband and intraband contributions, $\chi=\chi_{\text {intra }}+\chi_{\text {inter }}$. At low temperatures and finite occupancy at the Fermi level, such as in a metal, the intraband contribution normally dominates the exchange interactions. This part of the interaction is proportional to $\partial n(\varepsilon) / \partial \varepsilon$, where $n(\varepsilon)$ is the Fermi-Dirac distribution function of the energy band $\varepsilon$. At low temperature, this term converges to a delta-Dirac form and the intraband part of the susceptibility $\chi_{\text {intra }}$ is thus given primarily by the electrons around the Fermi level. Then, the indirect magnetic coupling can be well described in terms of the itinerant electrons of the Fermi surface, giving rise to a (generalized) RKKY interaction. By contrast, as long as the chemical potential lies within the energy gap of the ultrathin topological insulator film, the intraband contribution vanishes due to the absence of carriers. Hence, only interband contributions are now present, although they are also small [33]. These contributions, as their name indicate, come from processes between different bands and they become proportional to a term of the form $\left(n\left(\varepsilon_{1}\right)-n\left(\varepsilon_{2}\right)\right) /\left(\varepsilon_{1}-\varepsilon_{2}\right)$, where $\varepsilon_{1,2}$ indicates the two relevant bands in the system, normally valence and conduction bands. The part of the response function which originates from such interband processes between conduction and valence bands is usually referred to as a van Vleck interaction [19]. As this interaction is the response coming from different bands, separated by a gap, it decays exponentially with the size of the gap between the two bands [30]. In this work, since we also consider the effect of impurity states, the resulting complicated form of Eq. (7) prevents us from an analytical expansion, however, we use the above physical descriptions to understand our results. 

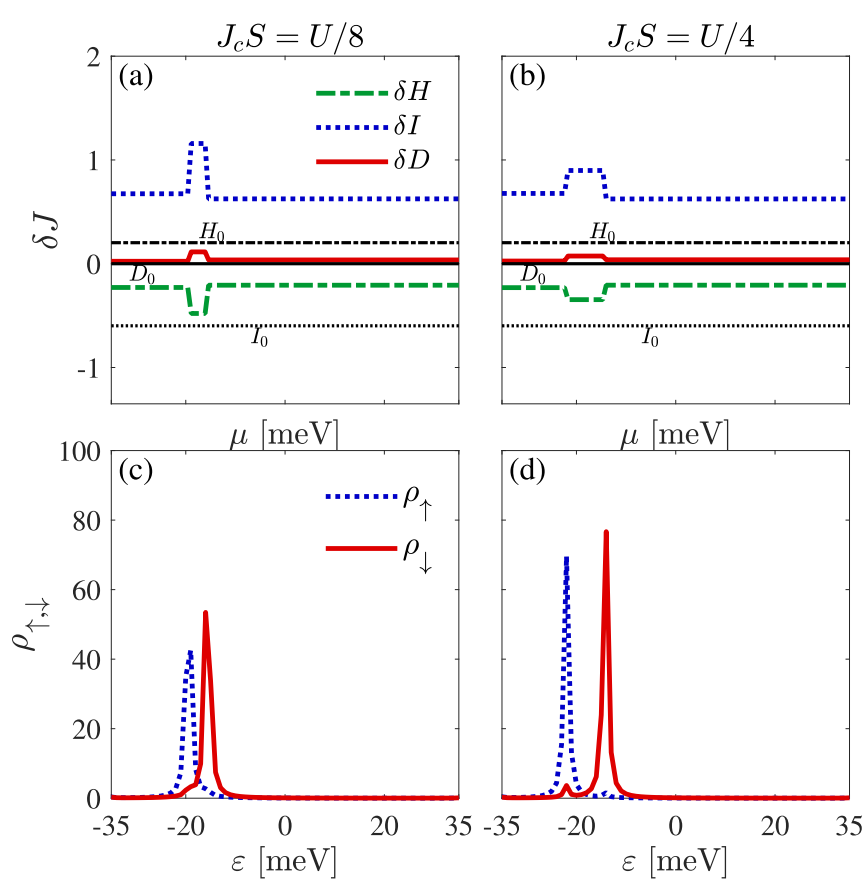

FIG. 1. Contributions of the exchange interaction, $\delta J=J-J_{0}$, where $J=H, I, D$, from the impurity states, as a function of chemical potential $\mu$. Here we assume two impurities located at distance $R=12.48 \AA$ and $V=0, U=4 \mathrm{eV}$, with $J_{c} S=U / 8$ (a) and $U / 4$ (b). Values for a pristine ultrathin topological insulator film is given by black lines for comparison. (c), (d) Corresponding spin-resolved LDOS with respect to the energy $\varepsilon$ in the presence of one impurity, with the LDOS calculated at the location of the other impurity.

\section{RESULTS}

Using Eq. (7) we quantify the complete and general indirect exchange coupling in terms of $J=H, I$, and $D$ between two magnetic impurities on the top surface of an ultrathin topological insulator film. We first present the different components in an unbiased $(V=0)$ film, and thereafter we discuss the effects of an external electric field. Building on these results, we proceed to study the magnetic ordering of impurity moments and the different magnetic phases in the system.

For the remainder of this work we assume a four quintuple layer thin film of $\mathrm{Bi}_{2} \mathrm{Se}_{3}$, which has an energy gap $2 \Delta=$ $70 \mathrm{meV}$ and Fermi velocity $v_{F}=4.48 \times 10^{5} \mathrm{~m} / \mathrm{s}$. We also assume that the chemical potential resides within the band gap, i.e., $|\mu|<\Delta$, in order to directly connect with recent experiments [10]. Moreover, and consistent with experiments, we assume an interimpurity distance of $R=12.48 \AA$ in the surface plane, unless we explicitly investigate the distance behavior. This choice is equivalent to $x=22 \%$ of $\mathrm{Cr}$ atoms in $\mathrm{Bi}_{2-x}(\mathrm{SeTe})_{3}$ used in recent experiments [2]. We also require that $0 \leqslant J_{c} S \leqslant 1$ and $0 \leqslant U \leqslant 6$ (both given in units of $\hbar v_{F} k_{c}$ ) and keep $J_{c} S / U \approx 1 / 8$ except Fig. 1(b) and 1(d) where we used $J_{c} S / U \approx 1 / 4$. We find these values by comparing our spin-resolved LDOS with the results of Refs. [36-39]. As we set the cutoff energy $\hbar v_{F} k_{c}$ to be $1 \mathrm{eV}$ in this work, all the presented $U, J_{c} S$ are in the unit of eV. We here note that the impurity potential terms and effective Hamiltonian of the topological insulator belong to two separate systems and the range of their validity should thus not be compared to each other. As impurity resonances are associated with local potentials, in some works even infinitely large values of $U$ have been considered in order to reproduce theoretically the experimental observations [24,40,41]. To simplify our plots we express the coupling terms, $J$, and spin-resolved LDOS in units of $\left(J_{c} / \hbar^{2} v_{F}^{2} \Omega_{\mathrm{BZ}}\right)^{2}$ and $1 / \hbar^{2} v_{F}^{2} \Omega_{\mathrm{BZ}}$, respectively, where $\Omega_{\mathrm{BZ}}$ is the area of the first Brillouin zone.

\section{A. Impurity states and their exchange interaction contributions}

We start by setting the parameters $V=0$ and $U=4 \mathrm{eV}$, in order to study how the impurities influence the exchange interaction. The plots in Fig. 1(a) and 1(b) show the corrections from the impurities, $\delta J=J-J_{0}$ with $J=H, D, I$ and $J_{0}=$ $H_{0}, I_{0}, D_{0}$, as a function of the chemical potential $\mu$ within the energy gap for $J_{c} S=U / 8$ and $J_{c} S=U / 4$, respectively. As a reference we also plot the exchange interactions parameters for the pristine topological insulator, $J_{0}$, with black lines. In Figs. 1(c) and 1(d) we show the corresponding spin-resolved LDOS in the presence of one impurity and calculated at the position of the second impurity as a function of energy $\varepsilon$ for the same values of $J_{c} S$ as panels (a) and (b), respectively, and chemical potential $\mu=0$.

First, we directly see that in the pristine case, the magnetic coupling is independent of the chemical potential, within the gap, in the pristine case. As the Fermi level lies within the gap of the system, the intraband part of the magnetic susceptibility, the RKKY interaction, is zero and the coupling can only come from the interband processes in the susceptibility. The interband processes are independent of the chemical potential, as long as the Fermi level is between the conduction and valence bands. These constant exchange interactions obtained for the impurity-free ultrathin topological insulator films should be contrasted with the interactions in the presence of impurities, which acquire both significantly different values and a very strong energy dependence, here reflected in the variation as a function of the chemical potential. In fact, we observe that the correction $\delta J$ to the exchange interaction changes the overall amplitude of the exchange coupling. From this observation, we conjecture that the carrier density redistributed from the valence band into the impurity resonances has a substantial overall influence on the magnetic susceptibility.

Specifically, we see that the isotropic and symmetrically anisotropic corrections $\delta H$ and $\delta I$ even obtain opposite signs compared to $H_{0}$ and $I_{0}$. On the other hand, the asymmetric anisotropy has significantly different behavior. Since the band dispersion of a pristine topological insulator film is electronhole symmetric, the overlap between states in the conduction and valence bands, due to their different helicities, result in a vanishing asymmetric anisotropy $D_{0}$ for all chemical potential values inside the gap. But, in the presence of the impurity resonances, the electron-hole symmetry is broken and the asymmetric anisotropy becomes finite.

There is also a very strong energy dependence for the exchange interactions in a specific range of the chemical potential. More specifically, whenever the chemical potential $\mu$ is positioned between the impurity resonances [see, e.g., the range $-20 \lesssim \mu \lesssim-15 \mathrm{meV}$ in Figs. 1(a) and 1(c)], the amplitudes of the magnetic coupling are strongly enhanced 

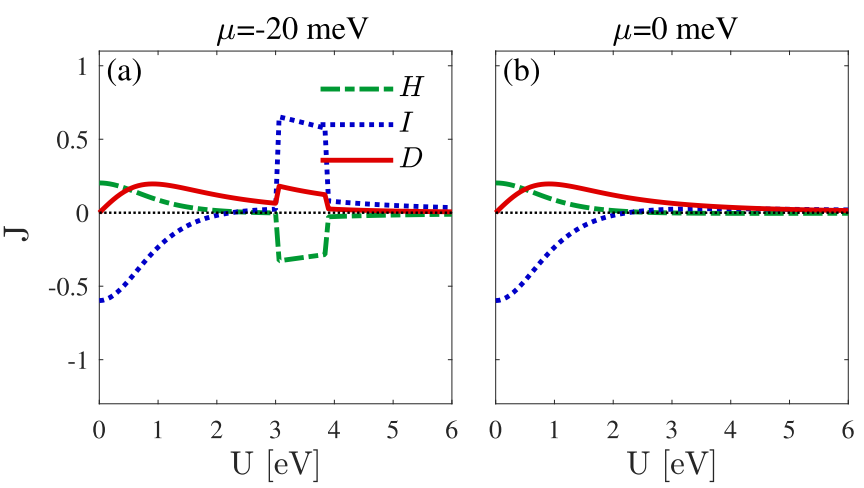

FIG. 2. Exchange interactions $J=H, I, D$ as a function of $U$ for $R=12.48 \AA, V=0$, and $J_{c} S=U / 8$ for $\mu=-20 \mathrm{meV}$ (a) and $\mu=$ $0 \mathrm{meV}$ (b) and with dotted black lines marking zero.

compared to when both impurity resonances lie on the same side of the chemical potential. This strong energy dependence can thus be directly traced back to the emergence of impurity resonances inside the band gap, as seen in Figs. 1(c) and 1(d). As a consequence of the finite $J_{c} S$, the impurity resonances are spin-split into two spin-polarized resonances [27]. These two resonances are very close to each other in energy and hence the interband interaction between them becomes very large. By increasing the value of $J_{c} S$ [panel (b)], the energy gap between the two impurity resonances becomes larger and the correction $\delta J$ thus becomes smaller. Moreover, as the energy gap between the impurity states and the valence band is smaller than the corresponding gap between these states and the conduction band, the correction $\delta J$ is slightly higher for energies smaller than the energy of impurity states.

Considering the properties of the impurities, both the spindependent $J_{c} S$ and spin-independent $U$ parts of the impurity potential can vary between the impurities. Therefore, in Fig. 2 we plot the exchange couplings $J=H, I, D$, as a function of the spin-independent impurity potential $U$, again keeping $R=12.48 \AA$ and $V=0$ and fixing $J_{c} S=U / 8$, for two different chemical potentials (a) $\mu=-20 \mathrm{meV}$ and (b) $\mu=$ $0 \mathrm{meV}$. In both cases we observe that the isotropic (symmetric anisotropic) exchange is positive (negative) for low scattering potentials but then transitions to negative (positive) values before diminishing for large $U$. The asymmetric anisotropy, on the other hand, vanishes in the absence of a scattering potential and peaks at small values before slowly approaching zero for increasing scattering potentials. However, in Fig. 2(a) this overall smooth dependence on $U$ is interrupted by a sharply defined region with larger values, in the range $U \sim 3 \mathrm{eV}$ to $U \sim 4 \mathrm{eV}$. These boundaries exactly mark the energies where at least one of the induced impurity resonances coincide with $\mu$, in analogy with the sharp features in Figs. 1(a) and 1(b). The substantially increased values of the exchange coupling in this $U$ range thus originate from that the impurity resonances residing within the band gap. The absence of the corresponding features in Fig. 2(b) is due to the fact that for this range of potentials $U$, the impurity resonances reside in the valence bands only and thus their effect is not present for the choice of in-gap value of $\mu$.
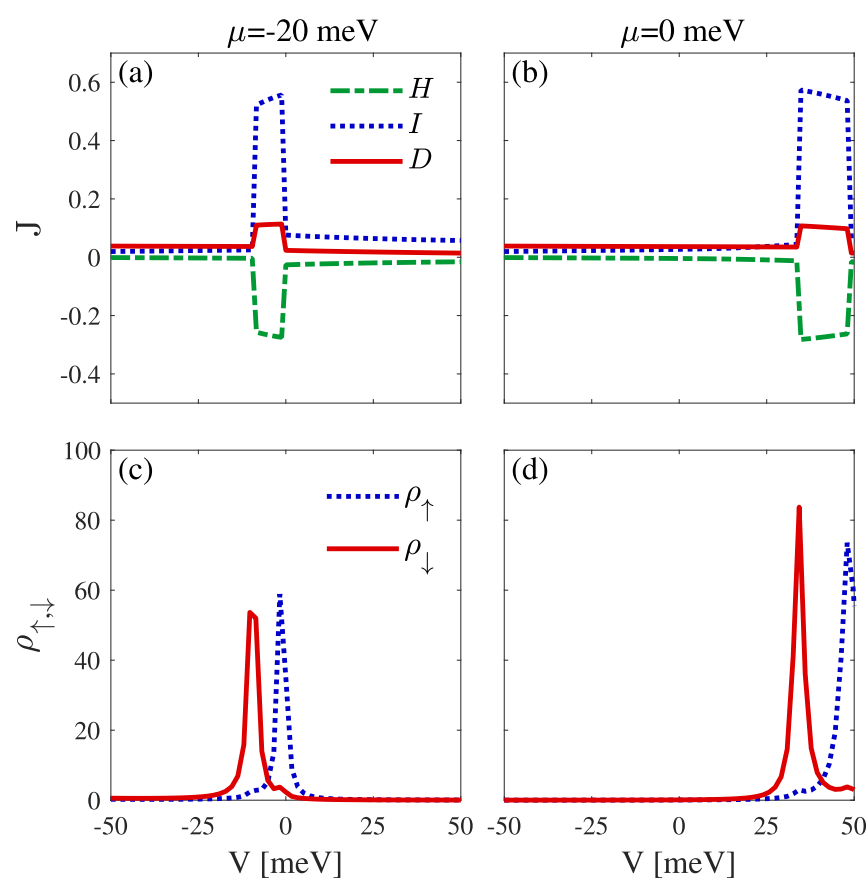

FIG. 3. Exchange interactions $J=H, I, D$ as a function of $V$ for $U=4 \mathrm{eV}, J_{c} S=U / 8, R=12.48 \AA$ and different chemical potentials $\mu=-20 \mathrm{meV}$ (a) and $\mu=0 \mathrm{meV}$ (b). (c), (d) Corresponding spin-resolved LDOS at energy $\varepsilon=\mu$ in for one impurity at the location of the other impurity.

\section{B. Giant electrical tunability of magnetism}

Having seen a strong dependence for the exchange coupling on the chemical potential, we next turn to the possibility to easily tune this behavior by applying an external electric field perpendicular to the plane of the film. We here choose the range between -50 and $50 \mathrm{meV}$ for the electrical potential drop as that is feasible to achieve for thin films a few nanometers thick [21]. To explore the signature of such potential difference in the magnetic exchange interaction, we present $H, I, D$ in terms of the parameter $V$ for different values of the chemical potential in Figs. 3(a) and 3(b), while in Figs. 3(c) and 3(d) we present the relevant spin-resolved LDOS extracted at energy $\varepsilon=\mu$ and plotted as a function of $V$. Clearly, we see how all the magnetic coupling terms are significantly higher for $V$ s between two distinct values. In between these two $V$ values the exchange coupling is in fact very large, for instance in (b) the Heisenberg coupling increases by $\sim 32$ times with respect to the unbiased case. For different chemical potentials the region of enhanced exchange coupling shifts, but it still exists equally prominently. The existence of a region with giant exchange couplings and its behavior with chemical potential and bias is explained by looking at the spin-resolved impurity resonance positions in Figs. 3(c) and 3(d). As has been shown before in Ref. [27], the application of an electric potential alters the position of impurities resonance peaks inside the gap, which then also moves the corresponding region with giant exchange couplings. Thus we find a giant electric tunability with an extreme sensitivity of the exchange interactions in an ultrathin topological insulator film. This giant tunability of the magnetic coupling is very 


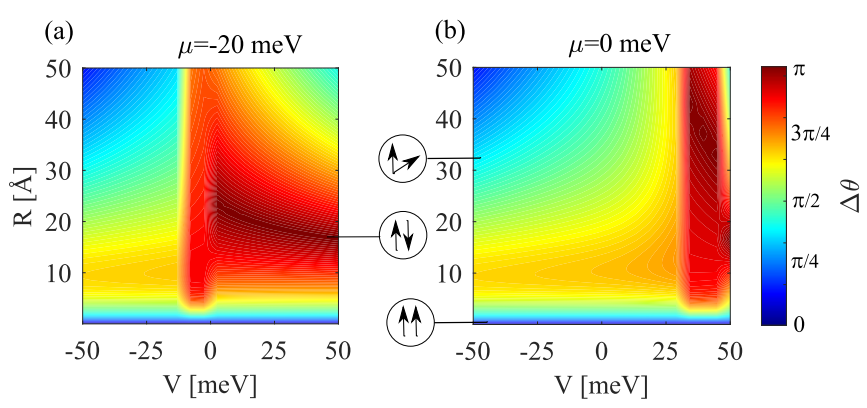

FIG. 4. Contour plots of energy-favorable angle between magnetic moments, $\Delta \theta$, in the plane of $R-V$ for two different chemical potentials (a) $\mu=-20 \mathrm{meV}$ and (b) $\mu=0$. Here we set $U=4 \mathrm{eV}$ and $J_{c} S=U / 8$. The relative angles of moments is illustrated by arrows at some points. Vertical axis in figure starts from $R=1 \AA$.

special to ultrathin TI films, as it is rooted in the tunable impurity resonances inside the gap. In most previous studies of electrical tunability, only around a few tens of percents have been reported $[21,32,33]$. It is worth mentioning here that qualitatively, all features exposed in Figs. 1-3 are also valid for other impurity distances (See Appendix F).

\section{Orientation of magnetic moments}

Having shown how two magnetic impurities have a highly unusual mutual interaction and also with a giant tunability, we next calculate the spin configuration of two magnetic moments. We continue to assume classical spins, which means that the Hamiltonian (6) can be rewritten as

$$
\begin{aligned}
\mathcal{H}^{\mathrm{ex}}= & |S|^{2}\left[(H+I) \cos \theta_{1} \cos \theta_{2}\right. \\
& +H\left(\cos \Delta \varphi+\cos \tilde{\varphi}_{1} \cos \tilde{\varphi}_{2}\right) \sin \theta_{1} \sin \theta_{2} \\
& \left.+D\left(\sin \theta_{1} \cos \theta_{2} \cos \tilde{\varphi}_{1}-\sin \theta_{2} \cos \theta_{1} \cos \tilde{\varphi}_{2}\right)\right],
\end{aligned}
$$

where $\theta_{1,2}$ and $\varphi_{1,2}$ are the polar and azimuthal angles of the spin vectors $\mathbf{S}_{1,2}$, respectively. Here, the azimuthal angles are considered with respect to $\varphi_{R}$, with $\tilde{\varphi}_{1(2)}=\varphi_{1(2)}-\varphi_{R}$ and we also define $\Delta \varphi=\varphi_{2}-\varphi_{1}$. Following straightforward calculations presented in Appendix C, we find that the minimum energy of two magnetic impurities coupled to each other is given by $\tan \Delta \theta=D /(H+J)$, where $\Delta \theta=\theta_{2}-\theta_{1}$ and $\varphi_{1}=\varphi_{2}=\varphi_{R}$. The nonalignment between the impurities is thus described in terms of the phase $\Delta \theta$. This phase is finite whenever the asymmetric anisotropy is finite $(D \neq 0)$ but vanishes in its absence. By introducing new spin variables $\bar{S}(\theta)$ with $|\bar{S}|=|\mathbf{S}|$, azimuthal angle $\varphi=\varphi_{R}$, and polar angle $\theta$, the effective spin Hamiltonian can be written as $H=\bar{S}(0) \cdot \bar{S}(\Delta \theta)$ for this arrangement [14].

To further investigate the spin configurations, we plot in Fig. 4 the relative polar angle $\Delta \theta$ between two magnetic moments, as a function of both the interimpurity distance $R$ and electric field $V$ for both $\mu=-20 \mathrm{meV}$ (a) and $\mu=0$ (b). In both cases the configuration tends towards becoming ferromagnetic, i.e., $\Delta \theta=0$, for very small distances between impurities and all values of $V$. At finite distances, however, the phase diagrams display a wide range of different configurations, spanning from ferromagnetic through noncollinear to antiferromagnetic configurations. In particular, at both chemical potentials distinctive regimes exist where the moments align toward an antiferromagneticlike configuration, $\Delta \theta>\pi / 2$, for all distances $R>10 \AA$ : in (a) $-10<V<$ $0 \mathrm{meV}$ and in (b) $35<V<50 \mathrm{meV}$. We trace these regimes directly back to the entry and exit of the chemical potential between the impurity resonances, see, e.g., Figs. 3(a) and 3(b). Beyond this electric field regime we find that most of the phase space consists of clearly noncollinear configurations, where the relative angle is both far from 0 and $\pi$, with the exact configuration determined by $R$. This is due to the large influence from the asymmetric anisotropy, which renders the collinear cases less favorable compared to a noncollinear arrangement.

Extending the results of Fig. 4 to a multi-impurity setup, we conclude that impurities favor pair configurations that can be represented by the angle $\Delta \theta$ in the $\rho z$ plane, where $\hat{\rho}$ defines the in-plane direction between impurities. Since the impurities are located in different directions $\hat{\rho}$, with respect to each other, the only common axis of all magnetic moments is along the $z$ axis. Hence, the resulting phase may most likely be ascribed a noncollinear ferromagnetic nature but with generally a $z$-axis component, i.e., an out-of-plane common component.

\section{CONCLUDING REMARKS}

In summary, we have investigated effects of impurity resonances on the magnetic exchange coupling between the magnetic moments located on the surface of ultrathin topological insulator films. We find that the contribution from the impurity resonances, typically, is of the same order as the bare contribution originating from the unperturbed surface states but become much larger under certain resonance conditions. We further analyze the importance of the impurity resonances on the magnetic interactions in terms of the isotropic, symmetric, and antisymmetric anisotropy components. For a pristine surface, the first two components are finite whereas the last one vanishes identically. We find that the contribution from the impurity states on the symmetric anisotropy and isotropic components, which both leads to collinear alignment of the magnetic moments, are within the same order of magnitude as the corresponding contributions from the intrinsic electronic structure, however, with opposite signs. Overall, this has a tendency to lead to a weakened collinear coupling. Most importantly, the noncollinear asymmetric anisotropic interaction, which is zero for pristine films, acquires a large contribution from the impurity states and imply that the collective ground state of the magnetic impurities should be strongly noncollinear.

Furthermore, we show that the applications of an electric field perpendicular to the ultrathin film can be used as a mechanism to shift the energy of the impurity resonances, which opens up the possibility to electrically tune the properties of the magnetic interactions. In effect, this mechanism should provide a tool for tuning between isotropic and anisotropic interactions, something which clearly has a great impact on the magnetic state.

Based on our findings we conclude that calculations of the magnetic exchange interactions, without considering effects originating from the impurities themselves are oversimplified 
[42]. Hence, there is a great risk of losing interesting and important features in the system. In particular, the exchange interaction based on the pristine topological insulator surface states misses the antisymmetric anisotropic component, which inevitable leads to the prediction of a noncollinear ferromagnetic phase. In fact, the easy axis of many magnetic impurities on the surface of topological insulators is in-plane [28], where calculations using the pristine system give in-plane ferromagnetic phase, while some experiments have already shown a perpendicular magnetic phase $[43,44]$. However, taking into account the influence from the impurity states but merely including the isotropic and symmetric anisotropic components of the exchange coupling $(H+I)$ does not provide a sufficient description, as it leads to an antiferromagnetic phase. Here, we have shown the importance of the antisymmetric anisotropy (D) term and the necessity to include it in calculations of the magnetic phase of ultrathin topological insulator films. It is this latter component (D) term that leads to a chiral ferromagnetic phase. Such chiral magnetic phase will clearly affect previous theoretical studies on QAHE experiments [45]. The QAHE has previously been assumed to be proportional to the net magnetization in the system. However, it has more recently been shown that the QAHE persists also in chiral ferromagnet and chiral antiferromagnetic systems with zero net magnetization [46], extending the effect to large parts of the phase diagram uncovered in this work.

\section{ACKNOWLEDGMENTS}

M.Sh. and J.F. thank the Carl Tryggers Stiftelse and Vetenskapsrådet for financial support. A.M.B.-S. and F.P. acknowledge financial support from the Swedish Research Council (Vetenskapsrådet Grant No. 2018-03488) and the Knut and Alice Wallenberg Foundation through the Wallenberg Academy Fellows program. M.Sh. also thanks the Institute for Research in Fundamental Sciences (IPM) for their hospitality.

\section{APPENDIX A: REAL SPACE GREEN'S FUNCTION}

In this Appendix we provide the components of the bare Green's function, i.e., for the pristine ultrathin topological insulator film without any impurities, which is used in the main text in Eq. (5) and also the onsite bare Green's function $g(\omega)$. The Matsubara Green's function in reciprocal space is given by $\mathcal{G}_{0}(\mathbf{k} ; \omega)=\left[i \omega+\mu-H_{0}\right]^{-1}$, which can be transformed into real-space by taking the Fourier transformation as

$$
\mathcal{G}_{0}(R ; \omega)=\frac{1}{\Omega_{\mathrm{BZ}}} \int d \mathbf{k} e^{i \mathbf{k} \cdot \mathbf{R}} \mathcal{G}_{0}(\mathbf{k} ; \omega) .
$$

After some straightforward calculations, the general matrix form of the bare Green's function is a $4 \times 4$ matrix reading

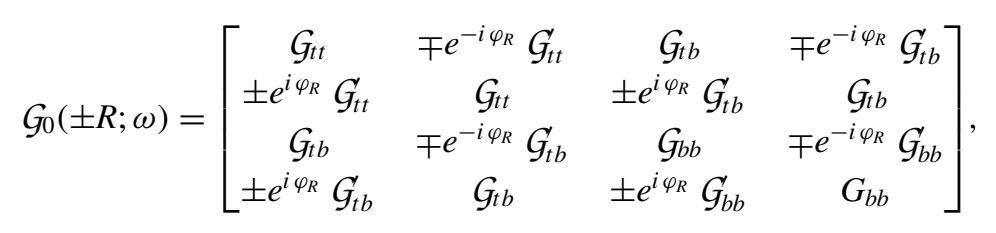

where $\varphi_{R}=\arctan \left(R_{y} / R_{x}\right)$ denotes the polar angle of the vector $\mathbf{R}=\mathbf{r}-\mathbf{r}^{\prime}$ between the two impurities. As we assume the impurities to be located on the top surface of the ultrathin topological insulator film, we need the upper right block of this matrix, in which $\mathcal{G}_{t t}$ and $\mathcal{G}_{t t}$ represent the $\uparrow \uparrow$ and $\uparrow \downarrow$ spin configurations of the bare Green's function, respectively, given by

$$
\begin{aligned}
& \mathcal{G}_{t t}(R ; \omega)=\frac{\pi}{\hbar^{2} v_{F}^{2} \Omega_{\mathrm{BZ}}} \sum_{s= \pm}\left(1+\frac{s \bar{\omega}}{\sqrt{\bar{\omega}^{2}-\Delta^{2}}}\right)\left(V-s \sqrt{\bar{\omega}^{2}-\Delta^{2}}\right) K_{0}(\tilde{R}) \\
& \mathcal{G}_{t t}(R ; \omega)=\frac{-i \pi}{\hbar^{2} v_{F}^{2} \Omega_{\mathrm{BZ}}} \sum_{s= \pm}\left(1+s \frac{\bar{\omega}}{\sqrt{\bar{\omega}^{2}-\Delta^{2}}}\right)\left|V-s \sqrt{\bar{\omega}^{2}-\Delta^{2}}\right| K_{1}(\tilde{R})
\end{aligned}
$$

where $\bar{\omega}=i \omega+\mu$ and $\tilde{R}=i R\left(V-s \sqrt{\bar{\omega}^{2}-\Delta^{2}}\right) / \hbar v_{F}$, and $K_{0,1}$ are the modified Bessel functions of the second kind. From these expressions we also find the analytic expression of the onsite Green's function

$$
\begin{aligned}
g= & \frac{\pi}{2 \hbar^{2} v_{F}^{2} \Omega_{\mathrm{BZ}}}\left[(V-\bar{\omega}) \ln \frac{\left(\left(\hbar v_{F} k_{c}\right)^{2}+V^{2}+\Delta^{2}-\bar{\omega}^{2}\right)^{2}-4\left(\hbar v_{F} k_{c} V\right)^{2}}{\left(V^{2}+\Delta^{2}-\bar{\omega}^{2}\right)^{2}}\right. \\
& \left.+2 \frac{V \bar{\omega}+\Delta^{2}-\bar{\omega}^{2}}{\sqrt{\Delta^{2}-\bar{\omega}^{2}}} \arctan 2 V^{2} \frac{\left(\hbar v_{F} k_{c}\right)^{2}-V^{2}+\Delta^{2}+\bar{\omega}^{2}}{\left(\Delta^{2}+\bar{\omega}^{2}\right)\left(\Delta^{2}+\bar{\omega}^{2}-2 V^{2}\right)}\right] .
\end{aligned}
$$

\section{APPENDIX B: SPIN-RESOLVED LDOS}

In this Appendix we provide the expression for the spin-resolved LDOS of an ultrathin topological insulator film in the presence of a single impurity. We use the $T$-matrix approach to find the dressed Green's functions. The $T$-matrix approach allows for a simple treatment of the scattering of surface Rashba-type electrons from a single impurity placed on the surface of the ultrathin topological insulator film. By considering a single impurity on the top surface including both an electrostatic and magnetic scattering potential as in Eq. (3), the LDOS for spin up and down electrons on the top surface of the total system can 
be obtained from the expression

$$
\boldsymbol{\rho}_{ \pm}=-\frac{1}{2 \pi} \operatorname{Im} \operatorname{tr}\left[\left(\sigma_{0} \pm \sigma\right) G(R ; \varepsilon)\right],
$$

where $G(R ; \varepsilon)$ refers to retarded Green's function, obtained from the Matsubara Green's function $\mathcal{G}(R ; \omega)$, by letting $i \omega \rightarrow$ $\varepsilon+i 0^{+}$. For a $\hat{z}$-axis polarized magnetic impurity, the spin-resolved LDOS is given by, see also Ref. [33],

$$
\begin{aligned}
& \rho^{\uparrow}=\frac{-1}{\pi} \operatorname{Im}\left[g+\frac{4 \pi^{2}\left(J_{c} S+U\right)}{g\left(J_{c} S+U\right)-1}\left(\sum_{s= \pm} s(V-i s \gamma) K_{0}(\tilde{R})\right)^{2}+\frac{4 \pi^{2}\left(J_{c} S-U\right)}{g\left(J_{c} S-U\right)+1}\left(\sum_{s= \pm} i a_{s} s(V-i s \gamma) K_{1}(\tilde{R})\right)^{2}\right], \\
& \rho^{\downarrow}=\frac{-1}{\pi} \operatorname{Im}\left[g+\frac{4 \pi^{2}\left(J_{c} S-U\right)}{g\left(J_{c} S-U\right)+1}\left(\sum_{s= \pm} s(V-i s \gamma) K_{0}(\tilde{R})\right)^{2}+\frac{4 \pi^{2}\left(J_{c} S+U\right)}{g\left(J_{c} S+U\right)-1}\left(\sum_{s= \pm} i a_{s} s(V-i s \gamma) K_{1}(\tilde{R})\right)^{2}\right] .
\end{aligned}
$$

In the above equations, $\gamma=\sqrt{\Delta^{2}-\left(\varepsilon+i 0^{+}\right)^{2}}$ and $a_{s}=\frac{1}{2}\left(\frac{\varepsilon+i 0^{+}}{\gamma}+i s\right)$, with the remaining quantities defined in the main text or in Appendix A.

\section{APPENDIX C: SPIN ORDERING}

In this Appendix we discuss in more detail how to find the minimizing condition for the relative spin configuration of two magnetic moments. Equation (8) in the main text is written in a rotated basis around the $\hat{z}$ direction by an angle $\varphi_{R}$, which makes the $\hat{x}$ axis along the direction between the two impurities. By applying another rotation of angle $-\pi / 2$ around the newly defined $\hat{x}$ axis, the $\hat{y}$ axis is interchanged with $\hat{z}: \hat{z} \rightarrow-\hat{y}$. Then in this new basis, the Hamiltonian is written as

$$
\begin{aligned}
\mathcal{H}^{\mathrm{ex}}= & |\mathbf{S}|^{2}\left(H \cos \theta_{1}^{\prime} \cos \theta_{2}^{\prime}+(H+I) \cos \left(\varphi_{1}^{\prime}-\varphi_{2}^{\prime}\right) \sin \theta_{1}^{\prime}\right. \\
& \left.\times \sin \theta_{2}^{\prime}+D \sin \left(\varphi_{1}^{\prime}-\varphi_{2}^{\prime}\right) \sin \theta_{1}^{\prime} \sin \theta_{2}^{\prime}\right),
\end{aligned}
$$

where $\theta^{\prime}$ and $\varphi^{\prime}$ are the polar and azimuthal angles, respectively, of the spin vectors in the new basis. The benefit of working in this new basis is that the Hamiltonian is dependent only on three angles as it is only related to the difference between azimuthal angles, $\phi=\varphi_{1}^{\prime}-\varphi_{2}^{\prime}$. By minimizing the Hamiltonian with respect to these three angles we find that the extrema of the system occur either at one of the five following points: $\left(\theta_{1}^{\prime}, \theta_{2}^{\prime}\right)=,(0,0),(0, \pi),(\pi, 0),(\pi, \pi)$, or $(\pi / 2, \pi / 2)$. For the first four points the azimuthal angles are not well defined and the Hessian matrix, which defines the concavity of the system, is zero and hence the system is at a saddle point. At the last point, $\left(\theta_{1}^{\prime}, \theta_{2}^{\prime}\right)=(\pi / 2, \pi / 2)$, the minimization condition occurs for the relative azimuthal angle $\phi=\arctan (D /(H+I))$. For a true minimum the determinant of the Hessian matrix, given by

$$
\mathcal{D}=-x\left(x^{2}-J^{2}\right)>0, \quad x=(H+I) \cos \phi+D \sin \phi,
$$

should be positive $[31,47]$. With two $\phi$ s satisfying the relation $\tan \phi=D /(H+I)$, we take the solution that makes $\mathcal{D}$ positive and arrive at the minimum energy configuration.

\section{APPENDIX D: EFFECT OF $\delta_{1} k^{2}$ TERM}

The model describing the hybridization between the two surfaces of an ultrathin topological insulator film has a general $k$-dependent form, $\Delta=\delta_{0}-\delta_{1} k^{2}$. The $k$-dependent $\delta_{1} k^{2}$ term is responsible for interesting topological effects $[34,48]$. However, in the two-dimensional infinite limit without considering any edges, we expect that this term does not change the qualitative picture of our results. Hence, for the sake of simplicity and to be able to achieve at least some analytical results for the real-space Green's function, we use in the main text of this work a hybridization parameter between the surfaces that is only consisting of the $k$-independent constant $\Delta=\delta_{0}$. In this Appendix we further justify our choice and show how our results must be in agreement with the result for $k$-dependent $\Delta=\delta_{0}-\delta_{1} k^{2}$ hybridization parameter. Figure 5 shows the total density of states of an ultrathin topological insulator film as a function of energy for two choices of hybridization parameter; $k$-independent $\Delta=\delta_{0}$ and $k$-dependent $\Delta=\delta_{0}-\delta_{1} k^{2}$. The figure shows clearly both impurity resonances and their density of states essentially completely coincide with each other for these two models. Since the van-Vleck spin susceptibility is primarily sensitive to the energy of the impurity resonances, we thus expect that the $k$ dependence of the surface hybridization can only play a very minor role for the magnetic coupling. We also note that Ref. [35] have studied the optical conductivity response for ultrathin topological insulator films, i.e., a related response function, and there also concluded that the addition

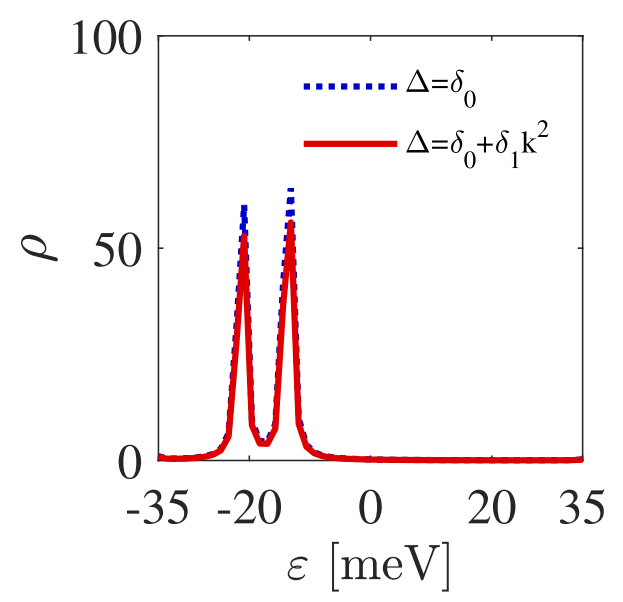

FIG. 5. Total density of states as a function of energy for two choices of hybridization parameter between the two topological insulator surfaces: $k$-independent $\Delta=\delta_{0}$ and $k$-dependent $\Delta=\delta_{0}-$ $\delta_{1} k^{2}$. Here we choose $\delta_{0}=35 \mathrm{meV}$ (as same as the main text) and $\delta_{1}=10 \mathrm{eVA}^{2}$ [11]. All other parameters are as same as Fig. 1(c). 

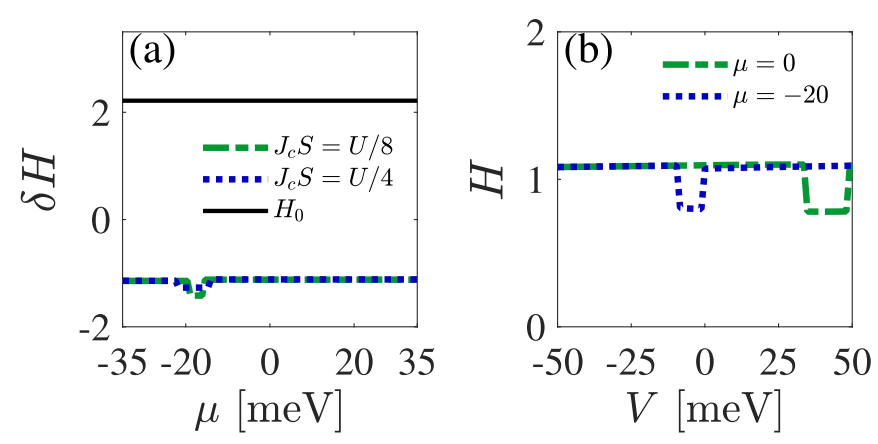

FIG. 6. (a) Heisenberg exchange interaction $H$ contribution from the impurity states, $\delta H=H-H_{0}$, as a function of chemical potential $\mu$ for two impurities located on different surfaces of an ultrathin topological insulator film, with zero in-plane distance for $V=0$, $U=4 \mathrm{eV}$, with $J_{c} S=U / 8, U / 4$. Response of the pristine ultrathin topological insulator film is illustrated with a black line for comparison. (b) Total exchange coupling $H$ with respect to potential $V$ for the same impurity configuration as in (a) at two chemical potentials $\mu=0,-20 \mathrm{meV}$. Here $U=4 \mathrm{eV}$ and $J_{c}=U / 8$.

of the $\delta_{1} k^{2}$ term does not change the qualitative behavior of the system response.

\section{APPENDIX E: INTERSURFACE COUPLING}

The ferromagnetic alignment of impurities in topological insulators have been experimentally observed for impurities located only on the top surface of topological insulator thin films $[5,49]$. This justifies the assumptions employed in this work, where we study the coupling of impurities located on only one of the surfaces. However, for the sake of completeness, and to also address other experiments for which impurities are located in all parts of the sample, we in this Appendix study the coupling of two impurities on opposite surfaces of the ultrathin topological insulator film.

In Fig. 6(a) we show the impurity contributions to the Heisenberg exchange coupling between two impurities located on different surfaces with a zero in-plane distance and for two choices of $J_{c} S$. For the sake of comparison, the contribution from a pristine topological insulator $H_{0}$ is also shown in black. The other two types of interactions, $D$ and $I$, are identically zero for the intersurface coupling between impurities. This can easily understood since, despite the strong spin-orbit coupling in TIs, the intersurface tunneling $\delta$ is spin degenerate and, hence, the form of intersurface exchange coupling reduces to a purely scalar, i.e. Heisenberg-like, interaction [33]. Beyond the $D$ and $I$ interactions being zero, we also see that the effect arising due to the impurities is more suppressed for intersurface Heisenberg exchange coupling than for the intrasurface exchange, although not entirely negligible. In Fig. 6(b) we present the variation of the Heisenberg term with respect to the potential $V$ at two different chemical potentials, $\mu=0$ and $-20 \mathrm{meV}$. This result suggests that the Heisenberg term is tunable by $\sim 20 \%$ by the potential $V$ and is of antiferromagnetic type. We note that the case of only Heisenberg-like interaction between the impurities on different surfaces technically breaks in the presence of Rashba splitting generated by $V$ [33]. However, for a chemical potential inside the gap, pertaining to this work, the Ising- and Dzyalosinskii-Moriya-like interactions are still negligible.

\section{APPENDIX F: INTERACTION AT LARGER DISTANCES}

In Figs. $1-3$ we focus on the exchange interaction at $R=$ $12.48 \AA$ A consistent with experiment [2]. Here, we present similar results but at the larger impurity-impurity distance, $R=$ $20 \AA$ A. In Fig. 7, the exchange interaction is plotted with respect to (a) the chemical potential $\mu$, (b) the spin-independent scattering potential $U$, and (c) the electric potential $V$. These plots show that the increased impurity-impurity distance does not change the qualitative behavior, but the results in the main text hold also at other larger distances. Specifically, the giant tunability of the interaction by an electric field, presented in panel (c) remains qualitatively the same. Here we note that, this larger distance $R=20 \AA$ corresponds to a density of $x=8 \%$ in $\mathrm{Bi}_{(2-x)} \mathrm{Cr}_{x}(\mathrm{SeTe})_{3}$, which is much lower than the $x=22 \%$ used in experiment to observe the QAHE [2]. Hence, studies of even larger distances between the impurities are not important for the purpose of the current work.
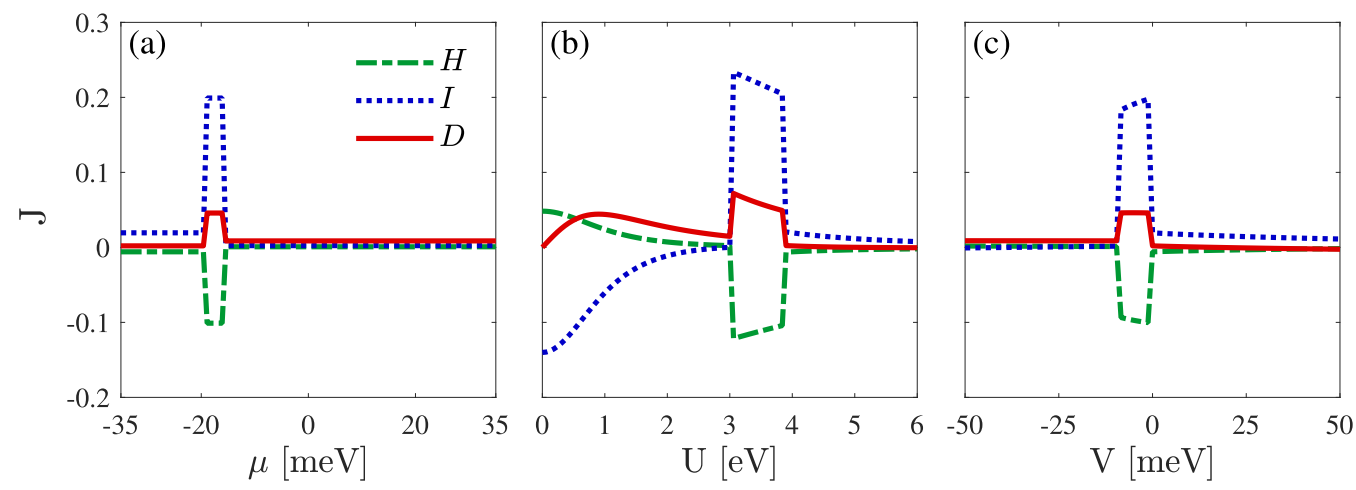

FIG. 7. Exchange interactions $J=H, I, D$ as a function of (a) chemical potential $\mu$, (b) impurity strength potential $U$, and (c) biased potential $V$ at a distance between impurities of $R=20 \AA$. Other parameters are: (a) $U=4 \mathrm{eV}, J_{c} S=U / 8, V=0$, (b) $\mu=-20 \mathrm{meV}, J_{c} S=$ $U / 8, V=0$, and (c) $\mu=-20 \mathrm{meV}, U=4 \mathrm{eV}, J_{c} S=U / 8$. 
[1] L. A. Wray, S.-Y. Xu, Y. Xia, D. Hsieh, A. V. Fedorov, Y. San Hor, R. J. Cava, A. Bansil, H. Lin, and M. Z. Hasan, A topological insulator surface under strong coulomb, magnetic and disorder perturbations, Nat. Phys. 7, 32 (2011)

[2] J. Zhang, C.-Z. Chang, P. Tang, Z. Zhang, X. Feng, K. Li, L.-1. Wang, X. Chen, C. Liu, W. Duan, K. He, Q.-K. Xue, $\mathrm{X}$. Ma, and Y. Wang, Topology-driven magnetic quantum phase transition in topological insulators, Science 339, 1582 (2013).

[3] Y. Okada, C. Dhital, W. Zhou, E. D. Huemiller, H. Lin, S. Basak, A. Bansil, Y.-B. Huang, H. Ding, Z. Wang, S. D. Wilson, and V. Madhavan, Direct Observation of Broken Time-Reversal Symmetry on the Surface of A Magnetically Doped Topological Insulator, Phys. Rev. Lett. 106, 206805 (2011).

[4] X. Kou, Y. Fan, M. Lang, P. Upadhyaya, and K. L. Wang, Magnetic topological insulators and quantum anomalous Hall effect, Solid State Commun. 215-216, 34 (2015).

[5] W. Liu, Y. Xu, L. He, G. van der Laan, R. Zhang, and K. Wang, Experimental observation of dual magnetic states in topological insulators, Sci. Adv. 5, eaav2088 (2019).

[6] C.-Z. Chang, J. Zhang, X. Feng, J. Shen, Z. Zhang, M. Guo, K. Li, Y. Ou, P. Wei, L.-L. Wang, Z.-Q. Ji, Y. Feng, S. Ji, X. Chen, J. Jia, X. Dai, Z. Fang, S.-C. Zhang, K. He, Y. Wang, L. Lu, X.-C. Ma, and Q.-K. Xue, Experimental observation of the quantum anomalous Hall effect in a magnetic topological insulator, Science 340, 167 (2013).

[7] C.-Z. Chang, W. Zhao, D. Y. Kim, H. Zhang, B. A. Assaf, D. Heiman, S.-C. Zhang, C. Liu, M. H. Chan, and J. S. Moodera, High-precision realization of robust quantum anomalous Hall state in a hard ferromagnetic topological insulator, Nat. Mater. 14, 473 (2015).

[8] J. Checkelsky, R. Yoshimi, A. Tsukazaki, K. Takahashi, Y. Kozuka, J. Falson, M. Kawasaki, and Y. Tokura, Trajectory of the anomalous Hall effect towards the quantized state in a ferromagnetic topological insulator, Nat. Phys. 10, 731 (2014).

[9] X. Kou, S.-T. Guo, Y. Fan, L. Pan, M. Lang, Y. Jiang, Q. Shao, T. Nie, K. Murata, J. Tang, Y. Wang, L. He, T.-K. Lee, W.-L. Lee, and K. L. Wang, Scale-Invariant Quantum Anomalous Hall Effect in Magnetic Topological Insulators Beyond the TwoDimensional Limit, Phys. Rev. Lett. 113, 137201 (2014).

[10] C.-X. Liu, S.-C. Zhang, and X.-L. Qi, The quantum anomalous Hall effect: Theory and experiment, Annu. Rev. Condens. Matter Phys. 7, 301 (2016).

[11] Y. Zhang, K. He, C.-Z. Chang, C.-L. Song, L.-L. Wang, X. Chen, J.-F. Jia, Z. Fang, X. Dai, W.-Y. Shan, S.-Q. Shen, Q. Niu, X.-L. Qi, S.-C. Zhang, X.-C. Ma, and Q.-K. Xue, Crossover of the three-dimensional topological insulator $\mathrm{Bi}_{2} \mathrm{Se}_{3}$ to the two-dimensional limit, Nat. Phys. 6, 584 (2010).

[12] H. Ohno, A window on the future of spintronics, Nat. Mater. 9, 952 (2010).

[13] D. K. Efimkin and V. Galitski, Self-consistent theory of ferromagnetism on the surface of a topological insulator, Phys. Rev. B 89, 115431 (2014).

[14] H. Imamura, P. Bruno, and Y. Utsumi, Twisted exchange interaction between localized spins embedded in a one- or two-dimensional electron gas with Rashba spin-orbit coupling, Phys. Rev. B 69, 121303 (2004).

[15] J. Fransson, Dynamical exchange interaction between localized spins out of equilibrium, Phys. Rev. B 82, 180411(R) (2010).
[16] M. Shiranzaei, J. Fransson, H. Cheraghchi, and F. Parhizgar, Nonlinear spin susceptibility in topological insulators, Phys. Rev. B 97, 180402(R) (2018).

[17] D. A. Abanin and D. A. Pesin, Ordering of Magnetic Impurities and Tunable Electronic Properties of Topological Insulators, Phys. Rev. Lett. 106, 136802 (2011).

[18] M. Zare, F. Parhizgar, and R. Asgari, Topological phase and edge states dependence of the RKKY interaction in zigzag silicene nanoribbon, Phys. Rev. B 94, 045443 (2016).

[19] R. Yu, W. Zhang, H.-J. Zhang, S.-C. Zhang, X. Dai, and Z. Fang, Quantized anomalous Hall effect in magnetic topological insulators, Science 329, 61 (2010).

[20] M. Li, C.-Z. Chang, L. Wu, J. Tao, W. Zhao, M. H. W. Chan, J. S. Moodera, J. Li, and Y. Zhu, Experimental Verification of the Van Vleck Nature of Long-Range Ferromagnetic Order in the Vanadium-Doped Three-Dimensional Topological Insulator $\mathrm{Sb}_{2} \mathrm{Te}_{3}$, Phys. Rev. Lett. 114, 146802 (2015).

[21] J. Wang, B. Lian, and S.-C. Zhang, Electrically Tunable Magnetism in Magnetic Topological Insulators, Phys. Rev. Lett. 115, 036805 (2015).

[22] A. Singh, A. Datta, S. K. Das, and V. A. Singh, Ferromagnetism in a dilute magnetic semiconductor: Generalized RKKY interaction and spin-wave excitations, Phys. Rev. B 68, 235208 (2003).

[23] J. Bouaziz, M. dos Santos Dias, A. Ziane, M. Benakki, S. Blügel, and S. Lounis, Chiral magnetism of magnetic adatoms generated by Rashba electrons, New J. Phys. 19, 023010 (2017).

[24] R. R. Biswas and A. V. Balatsky, Impurity-induced states on the surface of three-dimensional topological insulators, Phys. Rev. B 81, 233405 (2010).

[25] A. M. Black-Schaffer and A. V. Balatsky, Strong potential impurities on the surface of a topological insulator, Phys. Rev. B 85, 121103 (2012).

[26] A. M. Black-Schaffer, A. V. Balatsky, and J. Fransson, Filling of magnetic-impurity-induced gap in topological insulators by potential scattering, Phys. Rev. B 91, 201411 (2015).

[27] M. Shiranzaei, F. Parhizgar, J. Fransson, and H. Cheraghchi, Impurity scattering on the surface of topological-insulator thin films, Phys. Rev. B 95, 235429 (2017).

[28] M. F. Islam, C. M. Canali, A. Pertsova, A. Balatsky, S. K. Mahatha, C. Carbone, A. Barla, K. A. Kokh, O. E. Tereshchenko, E. Jiménez, N. B. Brookes, P. Gargiani, M. Valvidares, S. Schatz, T. R. F. Peixoto, H. Bentmann, F. Reinert, J. Jung, T. Bathon, K. Fauth, M. Bode, and P. Sessi, Systematics of electronic and magnetic properties in the transition metal doped $\mathrm{Sb}_{2} \mathrm{Te}_{3}$ quantum anomalous Hall platform, Phys. Rev. B 97, 155429 (2018).

[29] J. Sun, L. Chen, and H.-Q. Lin, Spin-spin interaction in the bulk of topological insulators, Phys. Rev. B 89, 115101 (2014).

[30] E. Kogan, RKKY interaction in gapped or doped graphene, Graphene 2, 8 (2013).

[31] F. Parhizgar, H. Rostami, and R. Asgari, Indirect exchange interaction between magnetic adatoms in monolayer $\mathrm{MoS}_{2}$, Phys. Rev. B 87, 125401 (2013).

[32] F. Parhizgar, M. Sherafati, R. Asgari, and S. Satpathy, Ruderman-Kittel-Kasuya-Yosida interaction in biased bilayer graphene, Phys. Rev. B 87, 165429 (2013).

[33] M. Shiranzaei, H. Cheraghchi, and F. Parhizgar, Effect of the Rashba splitting on the RKKY interaction in topologicalinsulator thin films, Phys. Rev. B 96, 024413 (2017). 
[34] W.-Y. Shan, H.-Z. Lu, and S.-Q. Shen, Effective continuous model for surface states and thin films of three-dimensional topological insulators, New J. Phys. 12, 043048 (2010).

[35] F. Parhizgar, A. G. Moghaddam, and R. Asgari, Optical response and activity of ultrathin films of topological insulators, Phys. Rev. B 92, 045429 (2015).

[36] J. Honolka, A. A. Khajetoorians, V. Sessi, T. O. Wehling, S. Stepanow, J.-L. Mi, B. B. Iversen, T. Schlenk, J. Wiebe, N. B. Brookes, A. I. Lichtenstein, P. Hofmann, K. Kern, and R. Wiesendanger, In-Plane Magnetic Anisotropy of Fe Atoms on $\mathrm{Bi}_{2} \mathrm{Se}_{3}$ (111), Phys. Rev. Lett. 108, 256811 (2012).

[37] T. Eelbo, M. Sikora, G. Bihlmayer, M. Dobrzański, A. Kozłowski, I. Miotkowski, and R. Wiesendanger, Co atoms on $\mathrm{Bi}_{2} \mathrm{Se}_{3}$ revealing a coverage dependent spin reorientation transition, New J. Phys. 15, 113026 (2013).

[38] T. Eelbo, M. Waśniowska, M. Sikora, M. Dobrzański, A. Kozłowski, A. Pulkin, G. Autès, I. Miotkowski, O. V. Yazyev, and R. Wiesendanger, Strong out-of-plane magnetic anisotropy of Fe adatoms on $\mathrm{Bi}_{2} \mathrm{Te}_{3}$, Phys. Rev. B 89, 104424 (2014).

[39] C. Zheng, Q. L. Li, B. F. Miao, L. Sun, R. Wang, X. X. Li, and H. F. Ding, Determining the strength of magnetic and potential scattering of magnetic impurities on the surface of a topological insulator via quantum corrals, Phys. Rev. B 96, 235444 (2017).

[40] T. O. Wehling, A. V. Balatsky, M. I. Katsnelson, A. I. Lichtenstein, K. Scharnberg, and R. Wiesendanger, Local electronic signatures of impurity states in graphene, Phys. Rev. B 75, 125425 (2007).

[41] N. M. R. Peres, F. Guinea, and A. H. Castro Neto, Electronic properties of disordered two-dimensional carbon, Phys. Rev. B 73, 125411 (2006).
[42] J.-J. Zhu, D.-X. Yao, S.-C. Zhang, and K. Chang, Electrically Controllable Surface Magnetism on the Surface of Topological Insulators, Phys. Rev. Lett. 106, 097201 (2011).

[43] Y. L. Chen, J.-H. Chu, J. G. Analytis, Z. K. Liu, K. Igarashi, H.-H. Kuo, X. L. Qi, S. K. Mo, R. G. Moore, D. H. Lu, M. Hashimoto, T. Sasagawa, S. C. Zhang, I. R. Fisher, Z. Hussain, and Z. X. Shen, Massive Dirac fermion on the surface of a magnetically doped topological insulator, Science 329, 659 (2010).

[44] C.-Z. Chang, J. Zhang, M. Liu, Z. Zhang, X. Feng, K. Li, L.-L. Wang, X. Chen, X. Dai, Z. Fang et al., Thin films of magnetically doped topological insulator with carrier-independent long-range ferromagnetic order, Adv. Mater. 25, 1065 (2013).

[45] K. He, Y. Wang, and Q.-K. Xue, Topological materials: Quantum anomalous Hall system, Annu. Rev. Condens. Matter Phys. 9, 329 (2018).

[46] H. Chen, Q. Niu, and A. H. MacDonald, Anomalous Hall Effect Arising from Noncollinear Antiferromagnetism, Phys. Rev. Lett. 112, 017205 (2014).

[47] R. A. Adams, Calculus: A Complete Course (Addison-Wesley, Toronto, 2003).

[48] F. Parhizgar and A. M. Black-Schaffer, Highly tunable timereversal-invariant topological superconductivity in topological insulator thin films, Sci. Rep. 7, 9817 (2017).

[49] T. Valla, Z.-H. Pan, D. Gardner, Y. S. Lee, and S. Chu, Photoemission Spectroscopy of Magnetic and Nonmagnetic Impurities on the Surface of the $\mathrm{Bi}_{2} \mathrm{Se}_{3}$ Topological Insulator, Phys. Rev. Lett. 108, 117601 (2012). 